de Andalucía (España). V. Acta Bot. Malacitana 26: 193-196.

CONDE, F., A. FLORES-MOYA, J. SOTO, M. ALTAMIRANO, \& A. SÁNCHEZ -1996aCheck-list of Andalusia (S. Spain) seaweeds. III. Rhodophyceae. Acta Bot. Malacitana 21: 7-33. CONDE, F., M. ALTAMIRANO y A. FLORESMOYA -1996b- Notas corológicas del macrofitobentos de Andalucía (España). IV. Acta Bot. Malacitana 21: 303-305.

FLORES-MOYA, A., J. SOTO, A. SÁNCHEZ, M. ALTAMIRANO, G. REYES \& F. CONDE -1995a- Check-list of Andalusia (S. Spain) seaweeds. I. Phaeophyceae. Acta Bot. Malacitana 20: 5-18.

FLORES-MOYA, A., J. SOTO, A. SÁNCHEZ, M. ALTAMIRANO, G. REYES \& F. CONDE -1995b- Check-list of Andalusia (S. Spain) seaweeds. II. Chlorophyceae. Acta Bot.
Malacitana 20: 19-26.

GUIRY, M. D. \& G. M. GUIRY -2009- AlgaeBase. World-wide electronic publication, National University of Ireland, Galway. http://www. algaebase.org; searched on 17 July 2009.

MAGGS, C. A., B. A. WARD, L. M. MCIVOR, C. M. EVANS, J. RUENESS \& M. J. STANHOPE 2002-Molecular analysis elucidate the taxonomy of fully corticated, nonspiny species of Ceramium (Ceramiaceae, Rhodophyta) in the British Isles. Phycologia 41: 409-420.

Dirección de los autores. ${ }^{1}$ Departamento de Biología Vegetal. Facultad de Ciencias. Universidad de Málaga. Campus de Teatinos, s/n. 29071, Málaga. ${ }^{2}$ Dpto. de Botánica. Facultad de Ciencias. Campus de Fuentenueva. Avda. Severo Ochoa s/n. 18071, Granada

*Autor para correspondencia: floresa@uma.es

\title{
182. NUEVAS CITAS DE PLANTAS ACUÁTICAS PARA LA LAGUNA REDONDA (MÁLAGA), RECIENTEMENTE RESTAURADA
}

\author{
Rafael Miguel CONDE-ÁLVAREZ ${ }^{* *}$, Félix LÓPEZ FIGUEROA², \\ José María NIETO³, José Miguel RAMÍREZ GONZÁLEZ4, \\ Fernando ORTEGA GONZÁLEZ y Manuel RENDÓN-MARTOS ${ }^{6}$.
}

Recibido el 1 de septiembre de 2009, aceptado para su publicación el 1 de octubre de 2009 Publicado "on line" en octubre de 2009

New record of aquatic plants from the "Laguna Redonda" (Málaga), a pond recently restored

Palabras clave. Plantas acuáticas, laguna, restauración de humedales, Laguna Redonda.

Key Word. Aquatic plants, pond, wetland restoration, Laguna Redonda.

La Laguna Redonda (30SUG3600) es una de las ocho lagunas pertenecientes al complejo lacustre "Lagunas de Campillos" situado en las Zonas externas de la Subbética (fig. 1). 

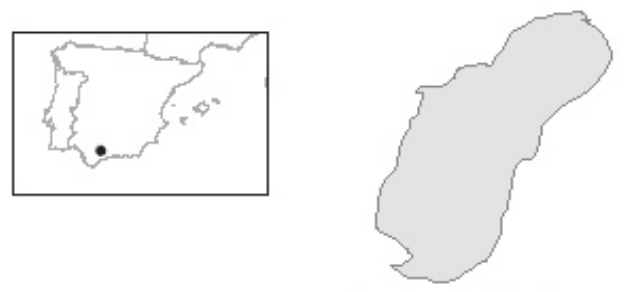

Fuente de Piedra

- Lobón

\section{Dulce}

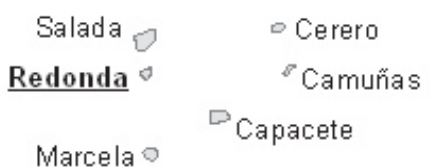

Figura 1. Situación de la Laguna Redonda.

Los materiales aflorantes están constituidos por depósitos triásicos, fundamentalmente litologías de naturaleza detrítico-yesífera, aunque también se entremezclan rocas carbonatadas.

La topografía del área donde se localizan las lagunas de Campillos es fundamentalmente llana y, en general, los suelos presentan un mal drenaje, con encharcamiento en periodo lluvioso. La escorrentía es de tipo difuso, produciendo fenómenos de erosión laminar que colmatan, aunque lentamente, las depresiones y empobrecen los suelos. Se trata de un área típicamente endorreica donde no existen corrientes superficiales y la erosión subterránea ha minado la superficie originando depresiones someras cerradas donde se alojan estas lagunas.

La Laguna Redonda es una pequeña laguna de tan solo 1,4 ha de cubeta y 28,19 ha de cuenca hidrológica. Sus aguas presentan un grado de mineralización dentro del rango de concentraciones hiposalinas, con una composición química clorurado-sulfatada sódico-cálcica. Todo el entorno de la laguna está altamente antropizado presentando cultivos herbáceos en secano y pastizales nitrófilos.

Esta laguna fue objeto de fuertes alteraciones antrópicas en las décadas de los años 70 y 80, al ser utilizada con cierta asiduidad para el depósito de escombros. La ocupación de la mayor parte del vaso lacustre por estos materiales, junto con las alteraciones de las escorrentías superficiales que alimentan la laguna ocasionó un comportamiento hídrico propio de un humedal temporal ocasional (Carrasco et al. 2005). Todas estas perturbaciones debieron tener repercusiones negativas en las comunidades acuáticas de la misma.

A principios de otoño de 2008 dentro del proyecto "Corrección de riberas y zonas húmedas en la provincia de Málaga", llevado a cabo por parte de la Consejería de Medio Ambiente de la Junta de Andalucía, se procedió a una serie de actuaciones en el vaso lagunar. De forma sintética, las actuaciones realizadas en el vaso lacustre consistieron en la retirada de escombros (13.304,70 $\mathrm{m}^{3}$ en total), y en la descompactación de la primera capa de sedimentos. Además, se procedió a la mejora de la red hídrica superficial, recuperando para la laguna sectores de la cuenca que antes eran drenados hacia los "Prados de Campillos". Con esta serie de actuaciones cabe esperar que la laguna funcione como un humedal estacional habitual, tal como parece indicar su comportamiento durante el presente año hidrológico.

Tras las actuaciones de restauración se comenzó a muestrear la laguna con una periodicidad mensual, en el marco del Plan Andaluz de Humedales y del proyecto ECOLIFE, tomándose datos de diferentes variables ambientales y de la presencia de plantas acuáticas (tab. 1).

Con las precipitaciones de otoño el vaso lacustre comienza a llenarse, alcanzándose el nivel más alto de agua en el mes de febrero (137 $\mathrm{cm})$, momento en el cual el agua presentaba una salinidad de $4,2 \mathrm{gl}^{-1}$.

A partir del mes de abril comienza a detectarse, la presencia de plantas acuáticas, 


\begin{tabular}{|c|c|c|c|c|c|}
\hline & $\begin{array}{l}\text { Mayo } \\
1997^{*}\end{array}$ & $\begin{array}{l}\text { Mayo } \\
\text { 1998* }\end{array}$ & $\begin{array}{l}\text { Abril } \\
2003^{*}\end{array}$ & $\begin{array}{l}\text { Mayo } \\
2008 \\
\end{array}$ & $\begin{array}{c}\text { Mayo } \\
2009 \\
\end{array}$ \\
\hline $\begin{array}{l}\text { Profundidad máxima } \\
(\mathrm{cm})\end{array}$ & $\approx 30$ & $\approx 60$ & 50 & $<50$ & 76 \\
\hline $\mathrm{pH}(\mathrm{U})$ & - & - & - & 7.52 & 9.17 \\
\hline $\begin{array}{l}\text { Temperatura del agua } \\
\left({ }^{\circ} \mathrm{C}\right)\end{array}$ & - & - & - & 18.7 & 19.5 \\
\hline Conductividad (mS/cm) & $\approx 6$ & $\approx 9$ & $\approx 31$ & 12.1 & 13.02 \\
\hline Salinidad (g/l) & $\approx 5$ & $\approx 5$ & $\approx 21$ & 6.9 & 7.5 \\
\hline Oxígeno disuelto (mg/l) & - & - & - & 4.07 & 6.92 \\
\hline $\begin{array}{l}\text { Vegetación sumergida } \\
\text { de fondo }(\%)\end{array}$ & - & - & - & 75 & 100 \\
\hline $\begin{array}{l}\text { Vegetación sumergida } \\
\text { flotante }(\%)\end{array}$ & - & - & - & 0 & $<10$ \\
\hline
\end{tabular}

Tabla 1.- Evolución de algunos de los parámetros controlados en el seguimiento de la Laguna Redonda. * Los datos relativos a estos años han sido tomados de la Caracterización ambiental de humedales en Andalucía (Moreira et al., 2004). Temporal variation of some parameters analyzed on the pond "Laguna Redonda". Data of years marked with asterisk symbols have been taken from Moreira et al. (2004).

presentándose a continuación el catálogo de las especies detectadas.

\section{CHARACEAE}

Chara aspera Detharding ex Wildenow 4753)

Conde-Álvarez et al.; 29-05-09 (MGC Algae

No se han encontrado referencias sobre la presencia de esta especie en la laguna en la bibliografía consultada, ni en la base de datos del proyecto GIBF. Dentro del complejo lacustre también se ha constatado la presencia de esta especie en la Laguna Salada (Asensi y Nieto, 1981), así como en la vecina laguna de Fuente de Piedra (Reyes Prósper, 1910; Asensi y Nieto, 1981).

\section{RIELLACEAE}

Riella helicophylla (Bory et Mont.) Mont.
Conde-Álvarez et al. (MGC Briof 1835).

No se han encontrado referencias sobre la presencia de esta especie en la laguna en la bibliografía consultada, ni en la base de datos del proyecto GIBF. En los muestreos realizados se detectó su presencia a partir del mes de abril, siendo de destacar que en el mes de mayo recubría prácticamente la totalidad del contorno de la laguna, formando en ciertos tramos densas praderas.

Dentro del complejo lacustre también se ha constatado su presencia en la Laguna Cerero (Cirujano et al., 1988; Conde-Álvarez et al. 1998), así como en la próxima laguna de Fuente de Piedra (Gil y Molero, 1984; Guerra et al. 1986; Cirujano et al., 1988; Cirujano et al., 1990; Conde-Álvarez, 2001).

\section{RANUNCULACEAE}

Ranuculus peltatus Schrank subsp peltatus Schrank. 
Conde-Álvarez et al. 29-05-09 (MGC 70343), previamente citado por Amat (1981).

\section{RUPPIACEAEE}

\section{Ruppia maritima L. var maritima}

Conde-Álvarez et al. 26-06-09 (MGC 70337), citada con anterioridad Furest et al. 1982 (SEV 87793) y citada por Cirujano \& García Murillo (1990).

\section{Ruppia drepanensis Tineo.}

Conde-Álvarez et al. (MGC 70338).

No se han encontrado referencias sobre la presencia de esta especie en la laguna en la bibliografía consultada, ni en las bases de datos de los proyectos Anthos y GIBF. En los muestreos realizados se detectó su presencia a partir del mes de abril.

Dentro del complejo lacustre también se ha constatado la presencia de esta especie en la Laguna Cerero (Cirujano et al., 1990), Laguna Marcela (Cirujano et al., 1990) y la Laguna Salada (Asensi y Nieto, 1981; Cirujano et al., 1990) así como en la cercana laguna de Fuente de Piedra (Cirujano et al., 1990), Conde-Álvarez 2001).

\section{ZANNICHELIACEAE}

Zannichellia obtusifolia Talavera, García Murillo \& Smit.

Conde-Álvarez et al. 26-06-09 (MGC 70344), ya citada por Talavera et al. (1986; SEV 101892) y Ortega (2002; JAEN 030309).

Por último, indicar que durante los muestreos realizados no se ha detectado la presencia de las siguientes especies de plantas acuáticas, citadas con anterioridad para esta laguna.

\section{CHARACEAE}

Tolypella hispanica Nordstdt ex T.F. Allen. Comelles (1982).

\section{POTAMOGETONACEAE}

\section{Potamogeton pectinatus L.} Amat (1981); García Murillo (1989).

\section{BIBLIOGRAFÍA}

AMAT, J. A. -1981- Ecología de las lagunas situadas en la periferia del Parque Nacional de Doñana. Fundación Juan March. Inenedit. 1-208.

ASENSI, A. y J. M. NIETO -1981- Vegetación acuática, halófila y halonitrófila de la provincia de Málaga. Trabajos y monografias del departamento de botánica de Málaga 2: 105-122.

CARRASCO, F., B. ANDREO, L. LINARES, M. RENDÓN, A. COBOS, F. ORTEGA, I. VADILLO e I. PÉREZ -2005-Contexto hidrogeológico de los humedales del norte de la provincia de Málaga. En: J. A. López-Geta, J. C. Rubio y M. Martín Machuca (Eds.), VI Simposio del Agua en Andalucía. IGME. pp. 605-618.

CIRUJANO, S. y P. GARCÍA MURILLO -1990Mapa 435 Ruppia maritima L. maritima en Fernández Casas, J. (ed.) Asientos para un atlas corológico de la Flora occidental. Fontqueria 28: 159-161.

CIRUJANO, S., C. MONTES, P. MARTINO, S. ENRÍQUEZ y GARCÍA MURILLO -1988Contribución al estudio del Género Riella Mont. (Sphaerocarpales, Riellaceae) en España. Limnetica 4: 41-50.

CIRUJANO, S., M. VELAYOS y C. GUERRERO 1990- Estudio de las zonas húmedas continentales españolas. Inventario, tipificación relación con el régimen hídrico general y medidas correctoras. Ministerio de obras públicas y Urbanismo. Manuscrito inéd. 048.

CONDE-ÁLVAREZR.M.,E. PEREZ-RODRIGUEZ, M. ALTAMIRANO, J. M. NIETO, R. ABDALA, F. L. FIGUEROA \& A. FLORES-MOYA-2002Photosynthetic performance and pigment content in the aquatic liverwort Riella helicophylla under natural solar irradiance and solar irradiance without ultraviolet light. Aquatic Botany 73 (1), 47-61.

CONDE-ÁLVAREZ, R. M. -2001- Variaciones espacio-temporales y ecofisiológicas de los macrófitos acuáticos de la laguna atalasohalina de Fuente de Piedra (Sur de la Península Ibérica). Tesis Doctoral. Universidad de Málaga.

GARCÍA MURILlO, P. J. -1990- El género Potamogeton L. en la Península Ibérica. Tesis doctoral. Universidad de Sevilla. 333 pp. 
GIL, J. A. Y J. MOLERO MESA -1984) Nueva cita de Riella helicophylla (Bory \& Mont.) para la Península Ibérica. Anal. Jard. Bot. Madrid 41 (1): 195-195.

GUERRA, J. E. RUIZ y C. SERGIO -1986- Sobre la distribución de Riella helicophylla (Bory et Mont.) Mont. en la Península Ibérica. Acta Bot. Malacitana 11: 75-76.

MOREIRA MADUEÑO, J. M (Edi.) -2004Caracterización ambiental de humedales en Andalucía. Consejería de Medio Ambiente. Sevilla ORTEGA, F., M. PARACUELLOS y F. GUERRERO -2004- Corología de macrófitos acuáticos en Andalucía oriental. Lazaroa 25: 179-185.

REYES PRÓSPER, E. -1910- Las carófitas de España. Singularmente las que crecen en sus estepas. Imprenta Artística Española. Madrid. $206 \mathrm{pp}$.

TALAVERA, S. P. GARCÍA MURILLO y H. SMIT -1986- Sobre el género Zannichellia L. (Zannichelliaceae). Lagascalia 14(2): 241-271.

Direcciones de los autores. ${ }^{1}$ Estudios de Flora y Vegetación (EFYVE) C/ Corregidor Nicolás $n^{\circ}$ 7, 29003 Málaga. ²Dpto. de Ecología y Geología, Univ.de Málaga, 29080 Málaga. ${ }^{3}$ Dpto. de Biología Vegetal, Univ. de Málaga, 29080 Málaga. ${ }^{4}$ EGMASA. P. C. Málaga Nostrum. Edif. Galia Center. C/ Jaén, 9-3 $3^{\text {a }}, 29004$ Málaga. ${ }^{5}$ Área de Ecología de la Univ. de Jaén. ${ }^{6}$ Consejería de Medio Ambiente, Junta de Andalucía. Oficinas de la Reserva Natural Laguna de Fuente de Piedra Cerro del Palo S/N 29520Fuente de Piedra (Málaga). *Autor para correspondencia: rconde@efyve.com.

\title{
183. NOVAS OCORRÊNCIAS DE SPHAGNUM AURICULATUM NO SUL DE PORTUGAL. SIGNIFICADO PALEOBIOGEOGRÁFICO
}

\author{
Carlos NETO $^{1 *}$, Pedro ARSÉNIO², Tiago MONTEIRO-HENRIQUES ${ }^{2}$, \\ Cecília SÉRGIO ${ }^{3}$ e José Carlos COSTA $^{1}$
}

Recibido el 21 de abril de 2009, aceptado para su publicación el 8 de junio de 2009 Publicado "on line" en junio de 2009

New chorological data of Sphagnum auriculatum in south Portugal. Paleobiogeographic meaning

Palavras-chave. Corologia, Sphagnum auriculatum, turfeiras de transição, sudoeste de Portugal.

Key words. Chorology, Sphagnum auriculatum, transition mires, southwestern Portugal.

O Sphagnum auriculatum Schimp. é uma espécie muito comum nas turfeiras baixas portuguesas e solos turfosos de linhas de água, especialmente no norte e centro de Portugal onde a precipitação média anual varia entre
$800 \mathrm{~mm}$ e $2800 \mathrm{~mm}$ (Séneca et al. 1992; Séneca, 1999; Séneca, 2003). No entanto, $S$. auriculatum perde em competição com outras espécies de musgos higrófilos (do mesmo género) nas turfeiras de altitude que tem muito 